\title{
Prediction of Clinically Relevant Herb-Drug Clearance Interactions Using Sandwich-Cultured Human Hepatocytes: Schisandra spp. Case Study
}

\author{
Jonathan P. Jackson, Kimberly M. Freeman, Weslyn W. Friley, Ashley G. Herman, \\ Christopher B. Black, Kenneth R. Brouwer, and Amy L. Roe \\ Qualyst Transporter Solutions, Durham, North Carolina (J.P.J., K.M.F., W.W.F., A.G.H., C.B.B., and K.R.B.) and Procter \& Gamble, \\ Cincinnati, Ohio (A.L.R.)
}

Received February 3, 2017; accepted June 29, 2017

\section{ABSTRACT}

The Schisandraceae family is reported to have a range of pharmacological activities, including anti-inflammatory effects. As with all herbal preparations, extracts of Schisandra species are mixtures composed of $>50$ lignans, especially schizandrins, deoxyschizandrins, and gomisins. In China, Schisandra sphenanthera extract (SSE) is often coadministered with immunosuppressant treatment of transplant recipients. In cases of coadministration, the potential for herb-drug interactions (HDIs) increases. Clinical studies have been used to assess HDI potential of SSE. Results demonstrated that chronic SSE administration reduced midazolam (MDZ) clearance by $52 \%$ in healthy volunteers. Although clinical studies are definitive and considered the "gold standard," these studies are impractical for routine HDI assessments. Alternatively, in vitro strategies can be used to reduce the need for clinical studies. Transporter-certified sandwich-cultured human hepatocytes ( $\mathrm{SCHHs)}$ provide a fully integrated hepatic cell system that maintains drug clearance pathways (metabolism and transport) and key regulatory pathways constitutive active/androstane receptor and pregnane $X$ receptor (CAR/PXR) necessary for quantitative assessment of HDI potential. Mechanistic studies conducted in SCHHs demonstrated that SSE and the more commonly used dietary supplement Schisandra chinensis extract (SCE) inhibited CYP3A4/5-mediated metabolism and induced CYP3A4 mRNA in a dose-dependent manner. SSE and SCE reduced MDZ clearance to 0.577 - and 0.599 -fold of solvent control, respectively, in chronically exposed $\mathrm{SCHHs}$. These in vitro results agreed with SSE clinical findings and predicted a similar in vivo HDI effect with SCE exposure. These findings support the use of an SCHH system that maintains transport, metabolic, and regulatory functionality for routine HDI assessments to predict clinically relevant clearance interactions.

\section{Introduction}

Conventional medicines share the same metabolic enzymes and xenobiotic transporters (e.g., cytochrome P450 enzymes, glucuronosyltransferases, P-glycoprotein, and organic ion transporters) that evolved in response to early human exposure to plant alkaloids and other phytochemicals. Thus, combinations of herbal ingredients with modern drugs may result in herb-drug interactions (HDIs) mediated by those metabolic enzymes and transporters. Interactions that increase plasma concentration or organ exposure of a drug may result in adverse drug events and significant toxicities, or conversely, therapies may fail when drug levels are reduced below minimal effective concentrations. The occurrence and significance of any specific HDI depends on a variety of

The laboratory research was funded by Qualyst Transporter Solutions and The Procter and Gamble Company, a research and development collaboration.

Some of the data in this work were presented as part of a poster abstract as follows: Jackson J, Freeman K, Friley W, Hipp K, Brouwer KR, and Roe AL. Evaluation of schisandra extract using sandwich-cultured human hepatocytes and B-CLEAR technology for the prediction of clinically relevant clearance interactions. 2016 Society of Toxicology Meeting; March 13-17 2016; New Orleans, LA.

https://doi.org/10.1124/dmd.117.075408. factors, such as dose, frequency and timing of herb/drug intake, dosing regimen, route of administration, and therapeutic range (Gurley, 2012; Sprouse and van Breemen, 2016).

In China, Schisandra sphenanthera extract (SSE) is prescribed as a tonic to improve hepatic function and is often coadministered with tacrolimus in treating renal and liver transplant recipients (Xin et al., 2007). In this case, any potential HDI may be life-threatening; therefore, it is important to predict these interactions. In recent human clinical studies, administration of SSE altered the first-pass metabolism, bioavailability, and plasma pharmacokinetics of tacrolimus (Qin et al., 2010). In similar studies, SSE increased the bioavailability and decreased the clearance of midazolam, with differential effects at the level of the gastrointestinal tract and liver (Xin et al., 2009). These data indicated clinically significant inhibitory effects on CYP3A4/5 in the presence of SSE.

Although clinical studies are definitive and considered the "gold standard," the number of combinations of herbal extracts and clinically used drugs is so large it is not feasible to routinely perform clinicalinteraction studies (Roe et al., 2016). Alternatively, in vitro strategies easily scale and can be used to reduce the need for clinical studies (Food and Drug Administration, 2006, 2012; European Medicines Agency, 2012; ). However, the accuracy of in vivo prediction from translation of

ABBREVIATIONS: DMSO, dimethylsulfoxide; HDI, herb-drug interaction; HPLC, high-pressure liquid chromatography; ICC, intracellular concentration; LC-MS/MS, liquid chromatography-tandem mass spectrometry; MDZ, midazolam; OHMDZ, 1-hydroxymidazolam; RIF, rifampicin; SCE, Schisandra chinensis extract; SCHH, sandwich-cultured human hepatocyte; SJW, St. John's wort; SSE, Schisandra sphenanthera extract; TAO, troleandomycin. 
in vitro results has been the "Achilles heel" of in vitro drug-interaction strategies. The choice of model system has been suggested to affect the prediction accuracy (McGinnity et al., 2005; Brown et al., 2007; Hewitt et al., 2007; Sprouse and van Breemen, 2016). For example, hepatic microsomes lack cellular processes including transporter (uptake and efflux) and adaptive response (e.g., induction) function. Therefore, microsomal studies cannot account for mechanisms that may affect unbound cellular concentrations in the hepatocyte. The lack of these processes likely decreases the accuracy of microsomes as an in vitro drug-drug interaction model.

Primary human hepatocytes have become the preferred in vitro system for transport, metabolism, toxicity, and drug-interaction studies due to their biologic (e.g., relevant species) and physiologic (e.g., wholecell model) relevance (Hewitt et al., 2007). Regulatory agencies, such as the Food and Drug Administration and European Medical Agency, have adopted primary human hepatocyte cultures as the standard in vitro model for predicting human cytochrome P450 enzyme induction by pharmaceuticals (European Medicines Agency, 2012; Food and Drug Administration, 2012). Under the proper culture conditions in conjunction with properly characterized human hepatocytes, a fully integrated hepatic cell system that maintains drug clearance pathways (metabolism and transport) and key regulatory pathways constitutive active/androstane receptor and pregnane $\mathrm{X}$ receptor $(\mathrm{CAR} / \mathrm{PXR})$ can be used to predictively screen for potential HDI.

Herein, we used a retrospective in vitro study design to evaluate the use of Transporter-Certified sandwich-cultured human hepatocytes (SCHHs) to predict in vivo HDI outcomes. Transporter-certified hepatocytes, which have been characterized for drug uptake and efflux transporter function while maintaining metabolism and nuclear receptor function, were used in this in vitro study to ensure hepatic physiologic relevance. SSE was used as a model herbal extract with proven clinical HDI while a second closely related and popular herbal dietary supplement extract, Schisandra chinensis extract (SCE), with an uncharacterized HDI profile was evaluated in parallel. Both mechanistic (e.g., inhibition and induction) and net effect (e.g., intrinsic clearance) studies were performed in SCHHs to demonstrate the effectiveness of this model to predict in vivo HDI outcomes.

\section{Materials and Methods}

\section{Materials}

Ketoconazole, aflatoxin, midazolam (MDZ), rifampicin (RIF), schisandrin A, gomisin A, schizandrin, d4-hydroxymidazolam, triazolam, dimethylsulfoxide (DMSO), $\beta$-mercaptoethanol, ammonium acetate, and formic acid were purchased from Sigma-Aldrich (St. Louis, MO). 1-Hydroxymidazolam (OHMDZ) was purchased from Cayman Chemical (Ann Arbor, MI). Troleandomycin (TAO) and d5-atorvastatin were purchased from Toronto Research Chemicals (Toronto, ON, Canada). High-pressure liquid chromatography (HPLC)-grade methanol and acetonitrile were purchased from World Wide Medical Products (Bristol, PA). Base cell culture media and other cell culture reagents were obtained from Thermo Fisher Scientific (Waltham, MA) and were of the highest purity available. St. John's wort (SJW) extract was purchased from PureBulk (Roseburg, OR). CellTiter-Glo Luminescent Cell Viability Assay was purchased from Promega (Madison, WI). Qiagen RNeasy kit and reagents were purchased from Qiagen (Germantown, MD). Quant-iT RiboGreen RNA Assay Kit, High Capacity cDNA Archive Kit, and TaqMan primer/probes were purchased from Thermo Fisher Scientific. Whatman 96-well Unifilter 25- $\mu \mathrm{m}$ melt blown polypropylene (MBPP) $0.45-\mu \mathrm{m}$ polypropylene (PP) filter plates, Millipore MDRPNP4 protein precipitation plate, and Millipore MSHVN45 $0.45-\mu \mathrm{m}$ filter plate were purchased from World Wide Medical Products.

Extracts of Schisandra fruit from both S. sphenanthera (SSE) and S. chinensis (SCE) were purchased from Flavex Naturextrakte GmbH (Rehlingen-Siersburg, Germany) and stored at room temperature prior to use. Extracts for both species were prepared from the dried fruit of Schisandra spp. by supercritical fluid extraction with natural carbon dioxide, with no solvent residues, no inorganic salts, no heavy metals, and no reproducible microorganisms. Thus, SSE and SCE contain all $\mathrm{CO}_{2}$-soluble lipophilic components, and both extracts have the appearance of a brown oily liquid with a weak aromatic smell. The SSE used in these studies contained $2.7 \%(\mathrm{~g} / 100 \mathrm{~g})$ deoxyschizandrin (also called schisandrin A) as detected by gas chromatography. The sum of schizandrins/gomisins (calculated as deoxyschizandrin) was 5.2\%. The SCE used in these studies contained $0.65 \%$ deoxyschizandrin (schisandrin A), $1.8 \%$ schizandrin, and $0.13 \%$ gomisin A, as detected by the supplier using gas chromatography. The sum of schizandrins/gomisins (calculated as deoxyschizandrin) was 7.2\%.

\section{Cell Culture}

SCHHs were prepared using QTS transporter-certified cryopreserved human hepatocytes (Table 1) purchased from Triangle Research Laboratories (Qualyst Transporter Solutions; Durham, NC). Cryopreserved hepatocytes were thawed following the manufacturer's thawing instructions. SCHHs were prepared by plating cryopreserved hepatocytes suspended in QualGro Seeding Medium, QTS propriety hepatocyte seeding medium, at a density of 0.8 million viable cells $/ \mathrm{ml}$ onto BioCoat 24-well cell culture plates purchased from Corning (Corning, NY), and maintained under standard cell culture conditions $\left(37^{\circ} \mathrm{C}, 5 \% \mathrm{CO}_{2}, 95 \%\right.$ humidity). Following plating, cells were allowed to attach for $2-4$ hours, rinsed, and fed with warm $\left(37^{\circ} \mathrm{C}\right)$ seeding medium. Eighteen to 24 hours later, cells were fed and overlaid with QualGro Hepatocyte Culture Induction Medium supplemented with $0.35 \mathrm{mg} / \mathrm{ml}$ Matrigel purchased from Corning. Cells were maintained in QualGro Hepatocyte Culture Induction Medium.

SSE and SCE Stock and Dosing Solution Preparation. SSE and SCE were provided as dark-yellow oils and diluted in DMSO to produce stock solutions, which were stored at room temperature. For chronic dosing studies (72 hours), SSE and SCE dosing solutions were prepared fresh daily in QualGro Hepatocyte Culture Induction Medium from these stock solutions, with final DMSO content $\leq 0.14 \%$. Treatment of hepatocyte cultures began on day 2 and continued for 72 hours. Each day, treatment solutions were removed, and fresh treatment solutions were added to hepatocytes. On day 5, MDZ intrinsic clearance assays were conducted, and cells were harvested for RNA isolation and intracellular concentration determinations.

Cytotoxicity Assessment. On day 5 of culture, viability of hepatocytes was quantitatively assessed by determining the amount of adenosine triphosphate (ATP) content present following 24 and 72 hours of exposure to increasing concentrations of SSE or SCE $(0.30,3.0,30$, and $300 \mu \mathrm{g} / \mathrm{ml}$ and $0.32,3.2,32$, and $320 \mu \mathrm{g} / \mathrm{ml}$, respectively). Aflatoxin $(10 \mu \mathrm{M})$ and media plus solvent $(0.14 \%$ DMSO) were included as positive and negative controls, respectively. Cellular ATP content was measured using CellTiter-Glo Luminescent Cell Viability Assay from Promega following the manufacturer's instructions. Luminescence detection of samples was measured using a SpectraMax GeminiXS 96-well Plate Reader (Molecular Devices, Sunnyvale, CA). Each test condition was performed in triplicate. The percentage of control was calculated as the ratio of ATP content in treated samples compared with that in wells treated with vehicle control $(0.14 \%$ DMSO) as described in eq. 1 :

$$
\% \text { of control }=\frac{\text { ATP content treatment }}{\text { ATP content } 0.1 \% \text { DMSO }} \times 100
$$

\section{CYP3A4/5 Enzyme Inhibition}

On day 5 of culture, direct and time-dependent inhibition of CYP3A4/5 in the presence and absence of SSE or SCE $(0.30,3.0,30$, and $300 \mu \mathrm{g} / \mathrm{ml}$ and 0.32, 3.2,

\section{TABLE 1}

Transporter Certified Cryopreserved Hepatocytes

\begin{tabular}{llll}
\hline Hepatocyte Lot & \multicolumn{1}{c}{ Sex } & \multicolumn{1}{c}{ Experiment } & \multicolumn{1}{c}{ Figure } \\
\hline HUM4059 & Female & 24-hour cytotoxicity assessment & Fig. 1A \\
HUM4075B & Male & 72-hour cytotoxicity assessment & Fig. 1B \\
HUM4059 & Female & CYP3A4/5 inhibition evaluation & Fig. 2 \\
HUM4109 & Female & CYP3A4 mRNA induction assessment & Fig. 3 \\
HUM4122C & Male & Intracellular concentration evaluation & Fig. 4 \\
HUM4122C & Male & MDZ intrinsic clearance studies & Figs. 5 and 6 \\
\hline
\end{tabular}


32 , and $320 \mu \mathrm{g} / \mathrm{ml}$, respectively) was assessed by monitoring the formation of OHMDZ. In parallel incubations, direct (50 and $250 \mathrm{nM}$ of ketoconazole) and time-dependent ( 1 and $10 \mu \mathrm{M} \mathrm{TAO}$ ) positive control inhibitors were evaluated as system controls. In brief, the medium of 5-day cultures of human hepatocytes was replaced with Williams' E medium containing $5 \mu \mathrm{M}$ MDZ with or without SSE, $\mathrm{SCE}$, or positive control inhibitor. Direct inhibition studies were conducted in situ at $37^{\circ} \mathrm{C}$ for 20 minutes. Following the conclusion of the incubation, medium was removed and stored at $-80^{\circ} \mathrm{C}$ prior to sample analysis. To investigate timedependent inhibition of CYP3A4/5, 5-day cultures of hepatocytes were incubated with SSE, SCE, or positive control for 60 minutes prior to addition of midazolam. After an additional 20-minute MDZ incubation period, medium was collected and stored at $-80^{\circ} \mathrm{C}$ prior to sample analysis. Samples and matrix-matched calibration standards were extracted (100- $\mu$ l sample volume) by the addition of $300 \mu l$ of internal standard solution (methanol containing $25 \mathrm{nM}$ triazolam) using a protein precipitation plate stacked on a 96-deep-well block. The plates were shaken prior to centrifugation for collection of the filtered supernatant. The sample filtrate was evaporated to dryness, and the samples were reconstituted in $200-\mu 1$ sample diluent, 40/60 methanol/10 mM ammonium acetate, and mixed for at least 20 minutes on a plate shaker. The reconstituted samples were transferred to a Millipore $0.45-\mu \mathrm{m}$ filter plate, filtered into a 96 -deep-well plate by centrifugation, and sealed with a silicone capmat. Liquid chromatography-tandem mass spectrometry (LC-MS/MS) analysis for the formation of the OHMDZ metabolite was performed using an API-3000 mass spectrometer (Applied Biosystems, Foster City, CA) operated in positive electrospray ionization mode. Chromatographic separation for OHMDZ was achieved using a binary HPLC system with LC-10ADvp pumps (Shimadzu, Columbia, MD) equipped with a Hypersil Gold $100 \times 1.0 \mathrm{~mm}, 3 \mu \mathrm{m}$ with matching guard and precolumn filter (Thermo Scientific, Bellefont, PA) using a linear gradient of 10\%-100\% B at a flow rate of $0.1 \mathrm{ml} / \mathrm{min}$, with mobile phase A as $10 \%$ methanol with $0.25 \mathrm{mM}$ ammonium acetate and mobile phase B as $80 \%$ methanol with $0.25 \mathrm{mM}$ ammonium acetate. Quantitation of OHMDZ was determined by HPLC-MS/MS monitoring for the mass spectrometry transition for OHMDZ $(\mathrm{m} / \mathrm{z} 342 \rightarrow 324)$. Calibration standards were prepared in blank media and extracted in parallel with study samples for quantitation of OHMDZ.

To quantify inhibition, percentage of remaining activity was calculated as shown in eq. 2 :

$$
\% \text { of remaining activity }=\frac{\text { OHMDZ formation of treated samples }}{\text { OHMDZ formation of solvent control }} * 100
$$

Nonlinear regression analysis of the inhibition dose-response curves (e.g., percentage of remaining activity versus test article concentration) was conducted using GraphPad Prism Software (version 6.0; GraphPad Software, La Jolla, CA). Estimated $\mathrm{IC}_{50}$ values and their respective $95 \%$ confidence intervals were determined using the nonlinear regression analysis functions within the software package. The strength of the parameter estimates was determined based upon the coefficient of determination $\left(R^{2}\right)$.

\section{CYP3A4 mRNA Induction}

SCHHs were treated with increasing concentrations of $\operatorname{SSE}$ or $\operatorname{SCE}(0.30,3.0$, 30 , and $300 \mu \mathrm{g} / \mathrm{ml}$ and $0.32,3.2,32$, and $320 \mu \mathrm{g} / \mathrm{ml}$, respectively) or with a clinically relevant CYP3A4 inducer, RIF $(10 \mu \mathrm{M})$, beginning on day 2 of culture and continuing for 72 hours. An extract of SJW $(20 \mu \mathrm{g} / \mathrm{ml})$, an herbal extract known to cause CYP3A4 induction, was also included for comparison. Following 72 hours of treatment (day 5 of culture), SCHHs were washed once with 1 volume of Hanks' balanced salt solution and lysed by addition of $0.3 \mathrm{ml}$ of Qiagen RLT Buffer supplemented with $\beta$-mercaptoethanol and frozen at $-80^{\circ} \mathrm{C}$. Lysates were thawed and total RNA was isolated from each treatment group, pooled from triplicate wells, using the Qiagen RNeasy kit following the manufacturer's instructions. RNA was stored at $-80^{\circ} \mathrm{C}$. Isolated RNA was quantified using Quant-iT RiboGreen RNA Assay Kit following the manufacturer's instructions. Pooled total RNA (500 ng) was converted to cDNA following the manufacturer's procedure for the High Capacity cDNA Archive Kit. CYP3A4 cDNA from human hepatocyte cultures was analyzed from each reverse transcription reaction using CYP3A4 (Hs00604506_m1) and glyceraldehyde-3-phosphate dehydrogenase (Hs99999905_m1) gene-specific TaqMan assays (Thermo Fisher Scientific). Glyceraldehyde-3-phosphate dehydrogenase was used as an endogenous control for gene-expression analysis. Amplifications were performed on a ViiA 7 RealTime PCR System (Thermo Fisher Scientific) in relative quantification mode for 45 amplification cycles using standard conditions for TaqMan-based assays. Threshold cycle $\left(\mathrm{C}_{\mathrm{T}}\right)$ determinations were performed by the ViiA 7 system software for both target and endogenous control genes. Relative-fold mRNA content was determined for each treatment group relative to the endogenous control gene expression and the calibrator, DMSO vehicle control, using the ViiA 7 system software. Confidence intervals of $95 \%$ were calculated for each target gene relative quantitation mean by the ViiA 7 system software.

Relative-fold mRNA content that was less than 1-fold, indicating suppression of gene expression, was transformed using eq. 3 to enhance the graphical representation of these data:

$$
\text { relative fold suppression }=\frac{-1}{\text { relative fold mRNA content }}
$$

\section{Intracellular Concentration of SSE and SCE Constituents}

The intracellular concentration (ICC) of three constituents of SSE and SCE (schisandrin A, gomisin A, and schizandrin) was determined in $\mathrm{SCHH}$ dosed with SCE or SSE at final assay concentrations of 30 and $300 \mu \mathrm{g} / \mathrm{ml}$ for 72 hours. Following 72 hours of treatment, cell culture medium was removed, and the tight junctions of treated SCHH were modulated using B-CLEAR technology (QTS; Triangle Research Laboratories) as previously described (Liu et al., 1999; Chandra and Brouwer, 2004; Ghibellini et al., 2007; Marion et al., 2012).

Cells were placed in frozen storage at $-80^{\circ} \mathrm{C}$ overnight prior to extraction, and quantitation of the constituents (schisandrin A, gomisin A, and schizandrin) was conducted. To extract, a volume of $500 \mu \mathrm{l}$ of lysis solution, 70:30 methanol:water (v:v) containing $100 \mathrm{nM} \mathrm{d5}$-atorvastatin (internal standard), was added to each well. Plates were shaken for at least 15 minutes, and the cell lysates were

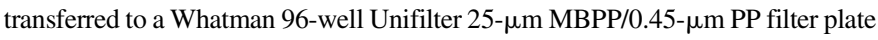
stacked on a 96-deep-well plate. Lysate was filtered into the deep-well plate by centrifugation. The sample filtrate was evaporated to dryness, and the samples were reconstituted in $150 \mu \mathrm{l}$ of sample diluent, $60 / 40$ methanol, and $10 \mathrm{mM}$ ammonium acetate and mixed for at least 10 minutes on a plate shaker. The reconstituted samples were transferred to a Millipore $0.45-\mu \mathrm{m}$ filter plate, filtered into a 96-deep-well plate by centrifugation, and sealed with a silicone capmat prior to LC-MS/MS analysis. LC-MS/MS analysis was performed using an Applied Biosystems API-3000 mass spectrometer operated in positive electrospray ionization mode. Chromatographic separation for schisandrin A, gomisin A, and schizandrin was achieved using a Shimadzu binary HPLC system with LC-10ADvp pumps equipped with a Thermo Scientific Hypersil Gold AQ column $(100 \times 1.0 \mathrm{~mm}, 3 \mu \mathrm{m})$ with a matching guard and precolumn filter using a linear gradient of $20 \%-100 \%$ B, with mobile phase A as $0.2 \%$ formic acid in water and mobile phase B as $0.2 \%$ formic acid in acetonitrile. Quantitation was determined by HPLC-MS/MS monitoring for the mass spectrometry transition for schisandrin A $(\mathrm{m} / \mathrm{z}, 417 \rightarrow 399)$, gomisin A $(\mathrm{m} / \mathrm{z}, 417 \rightarrow 399)$, and schizandrin $(\mathrm{m} / \mathrm{z}$ $433 \rightarrow 415$ ). Calibration standards were prepared using previously frozen $24-w e l l$ analytical plates derived from SCHH spiked with known concentrations of each constituent and extracted in parallel with unknown samples. Sample concentrations were reported as peak-area ratios back calculated from the calibration curve.

The ICC of each analyte was calculated by dividing the mass of analyte in the hepatocytes (cellular accumulation) by the hepatocyte intracellular fluid volume of $7.69 \mu 1 / \mathrm{mg}$ protein for hepatocytes, as previously described (Lee and Brouwer, 2010). All mass values were generated from triplicate wells and are represented by the calculated mean and S.D. from these biologic replicates.

\section{MDZ Intrinsic Clearance Assays}

Intrinsic clearance of $\mathrm{MDZ}$ was evaluated in $\mathrm{SCHH}$ on day 5 of culture following 72 hours of exposure to RIF $(10 \mu \mathrm{M})$, SJW $(20 \mu \mathrm{g} / \mathrm{ml})$, and SSE ( 3 and $30 \mu \mathrm{g} / \mathrm{ml}$ ) and SCE (3 and $30 \mu \mathrm{g} / \mathrm{ml}$ ). Twenty-four hours after last dosing, cell culture medium was aspirated and replaced with Williams' E medium containing MDZ $(1 \mu \mathrm{M})$. At multiple time points, incubations were terminated with the addition of $900 \mu \mathrm{l}$ of methanol containing $100 \mu \mathrm{M} \alpha$-hydroxymidazolam-d4 (internal standard) and frozen at $-80^{\circ} \mathrm{C}$ prior to analysis. The supernatants were removed, filtered, dried down under nitrogen, and reconstituted in $150 \mu \mathrm{l}$ of 60/40 methanol/water with $10 \mathrm{mM}$ ammonium acetate and analyzed for the loss of 
parent MDZ using an Applied Biosystems API-3000 mass spectrometer operated in positive electrospray ionization mode. Chromatographic separation for MDZ was achieved using a Shimadzu binary HPLC system with LC-10ADvp pumps equipped with a Thermo Scientific Hypersil Gold column $(50 \times 1.0 \mathrm{~mm}, 3 \mu \mathrm{m})$ with matching guard and precolumn filter using a linear gradient of $10 \%-100 \% \mathrm{~B}$, at a flow rate of $0.075 \mathrm{ml} / \mathrm{min}$, with mobile phase $\mathrm{A}$ as $0.2 \%$ formic acid in water and mobile phase B as $0.2 \%$ formic acid in methanol. Quantitation of MDZ was determined by HPLC-MS/MS monitoring for the mass spectrometry transition for $\operatorname{MDZ}(\mathrm{m} / 2,326 \rightarrow 248)$.

The substrate-depletion approach was used to estimate metabolic intrinsic clearance. Linear regression analyses of the percentage of parent remaining (logtransformed) versus time profiles were used to estimate the elimination rate constant $(\mathrm{k})$. Intrinsic clearance was estimated assuming monoexponential decay using eq. 4 :

$$
\text { Clearance }{ }_{\text {intrinsic }}=\mathrm{k}^{*} \mathrm{~V} / \mathrm{N}
$$

where $\mathrm{V}=$ total incubation volume, $\mathrm{N}=$ milligram of protein in the incubation, and $\mathrm{k}=$ elimination rate constant. The observed metabolic intrinsic clearance was scaled utilizing scaling factors derived from physiologic parameters for their respective species (Davies and Morris, 1993). To quantify the change in MDZ intrinsic clearance in response to treatment, percentage of control was calculated as the ratio of intrinsic clearance observed in treated samples to intrinsic clearance observed in vehicle control ( $0.14 \%$ DMSO) as described in eq. 5 :

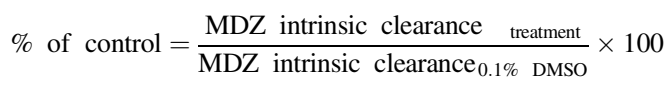

Statistical Analyses. All calculations were performed using Excel 2010 (Microsoft, Redmond, WA) unless otherwise stated. Statistical analysis was performed using GraphPad Prism Software (version 6.0). A one-way analysis of variance followed by a Dunnett's test was performed for statistical comparisons of ATP content and MDZ intrinsic clearance. An * in figures denotes a $P$ value $\leq 0.05$.

\section{Results}

Cytotoxicity Assessment. Intracellular ATP content was evaluated following 24 and 72 hours of treatment to assess cytotoxicity of Schisandra spp. extracts, SSE $(0.30,3.0,30.0$, and $300 \mu \mathrm{g} / \mathrm{ml})$ and $\operatorname{SCE}(0.32,3.2,32$, and $320 \mu \mathrm{g} / \mathrm{ml})$. The cellular ATP content in SCHHs was significantly $(P$ value $\leq 0.05$ ) reduced to $27.4 \%$ and $10.9 \%$ of solvent control following 24 and 72 hours, respectively, of exposure to aflatoxin $(10 \mu \mathrm{M})$ (Fig. 1). These results demonstrated that the SCHHs were sensitive to known metabolism-dependent toxins, such as aflatoxin (Essigmann et al., 1977). No marked reduction in SCHH cell viability was observed in SCHHs treated for 24 or 72 hours with $0.30,3.0$, or $30 \mu \mathrm{g} / \mathrm{ml} \mathrm{SSE}$ and $0.32,3.2$, or $32 \mu \mathrm{g} / \mathrm{ml} \mathrm{SCE}$ (Fig. 1). In contrast, ATP content was significantly $(P$ value $\leq 0.05)$ reduced to $86.5 \%$ and $89.7 \%$ of solvent control in SCHHs following 24 hours of treatment with $300 \mu \mathrm{g} / \mathrm{ml} \mathrm{SSE}$ and $320 \mu \mathrm{g} / \mathrm{ml} \mathrm{SCE}$ (Fig. 1A). Following 72 hours of treatment with $300 \mu \mathrm{g} / \mathrm{ml} \mathrm{SSE}$ and $320 \mu \mathrm{g} / \mathrm{ml} \mathrm{SCE}$, ATP content was significantly ( $P$ value $\leq 0.05$ ) reduced to $89.5 \%$ and $76.5 \%$ of solvent control in SCHHs (Fig. 1B). Overall, these results suggested that SSE and SCE were noncytotoxic at concentrations $\leq 30$ and $32 \mu \mathrm{g} / \mathrm{ml}$, respectively, following 24 or 72 hours of exposure.

CYP3A4/5 Inhibition Assessment. CYP3A4/5 activity was evaluated in the absence and presence of $\operatorname{SSE}(0.30,3.0,30.0$, and $300 \mu \mathrm{g} / \mathrm{ml})$ and SCE $(0.32,3.2,32$, and $320 \mu \mathrm{g} / \mathrm{ml})$ with and without preincubation to assess the inhibition potential of Schisandra spp. extracts. The rate of OHMDZ formation was significantly decreased approximately $28 \%$ or $72 \%$ when SCHHs were incubated in the presence of the known inhibitor ketoconazole (50 or $250 \mathrm{nM}$, respectively; data not shown). Similarly, inclusion of either SCE or SSE in the incubation medium significantly decreased the rate of OHMDZ formation in a
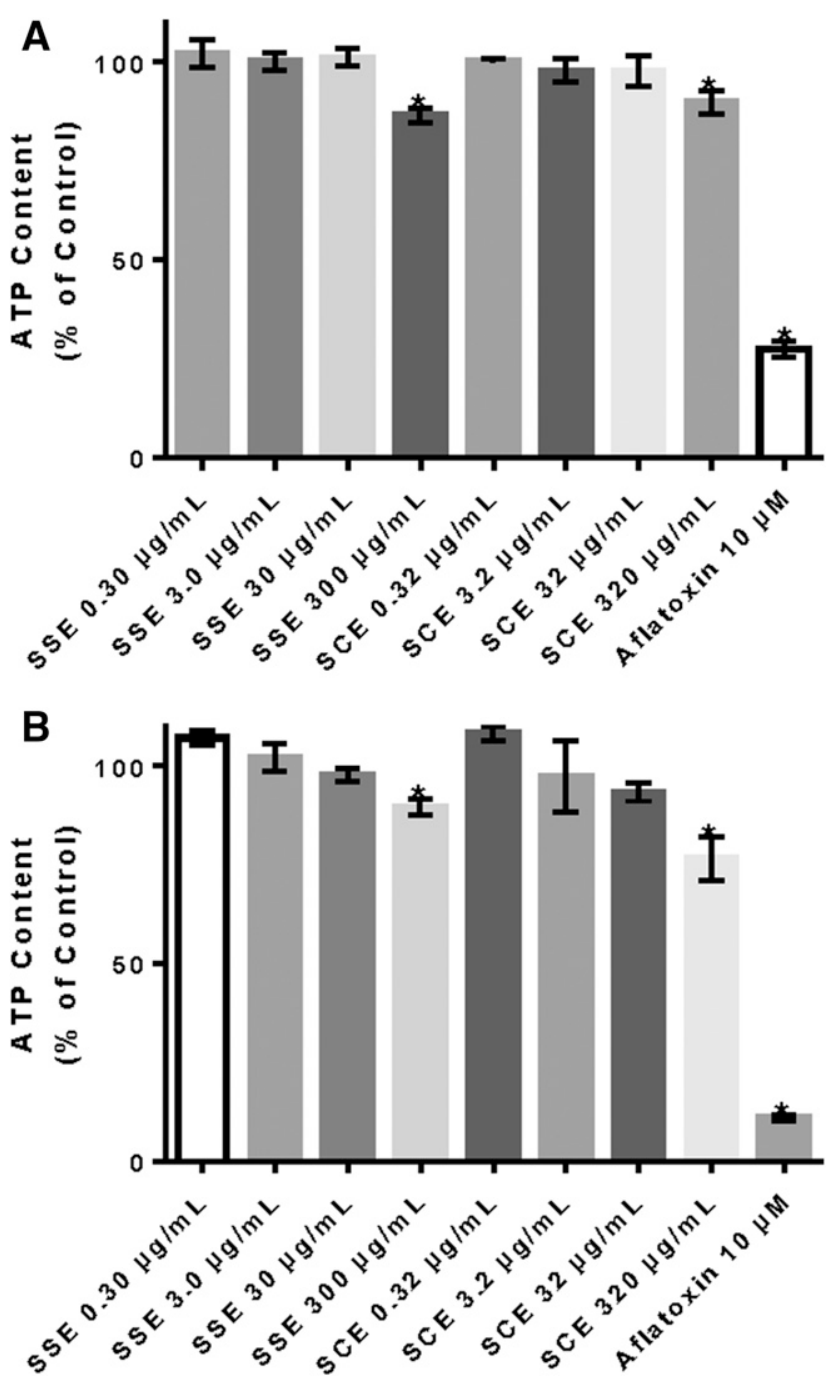

Fig. 1. ATP content evaluation in SCHH following 24- (A) and 72-hour exposure to varying concentrations of SSE and SCE. Aflatoxin $(10 \mu \mathrm{M})$ was used as a positive control cytotoxic agent. ${ }^{*} P$ value $\leq 0.05$ when compared with solvent control. Data are from a single experiment with treatments performed in triplicate wells. Mean values are reported \pm S.D.

concentration-dependent manner. In the absence of preincubation, CYP3A4 enzyme activity was reduced to $3.29 \%$ and $4.13 \%$ of solvent control at the highest SSE $(300 \mu \mathrm{g} / \mathrm{ml})$ and SCE $(320 \mu \mathrm{g} / \mathrm{ml})$ concentrations examined in SCHHs (Fig. 2). These results demonstrated that both Schisandra spp. extracts have the potential to inhibit CYP3A4/5 activity as direct inhibitors. Nonlinear regression analysis of the inhibition plots (percentage of remaining activity vs. the log concentration of SCE or SSE) estimated $\mathrm{IC}_{50}$ values of $4.46 \mu \mathrm{g} / \mathrm{ml}$ for SCE and $1.23 \mu \mathrm{g} / \mathrm{ml}$ for SSE.

When TAO $(1 \mu \mathrm{M})$ was added to wells 60 minutes prior to the addition of MDZ, the rate of OHMDZ formation by SCHH was reduced approximately $80 \%$ compared with a $20 \%$ reduction when TAO was not preincubated, thus demonstrating time-dependent inhibition by TAO (data not shown). SSE $(3 \mu \mathrm{g} / \mathrm{ml})$ and SCE $(3.2 \mu \mathrm{g} / \mathrm{ml})$ reduced the rate of OHMDZ formation to $18.6 \%$ and $24.6 \%$ of solvent control, respectively, in $\mathrm{SCHH}$ following a 60-minute preincubation (Fig. 2). Without preincubation, the OHMDZ formation rate by $\mathrm{SCHH}$ was reduced to $37.4 \%$ and $67.1 \%$ of solvent control (Fig. 2) in the presence of SSE $(3 \mu \mathrm{g} / \mathrm{ml})$ and SCE $(3.2 \mu \mathrm{g} / \mathrm{ml})$, correspondingly. Taken together, these results demonstrated that inhibition of OHMDZ formation in the 

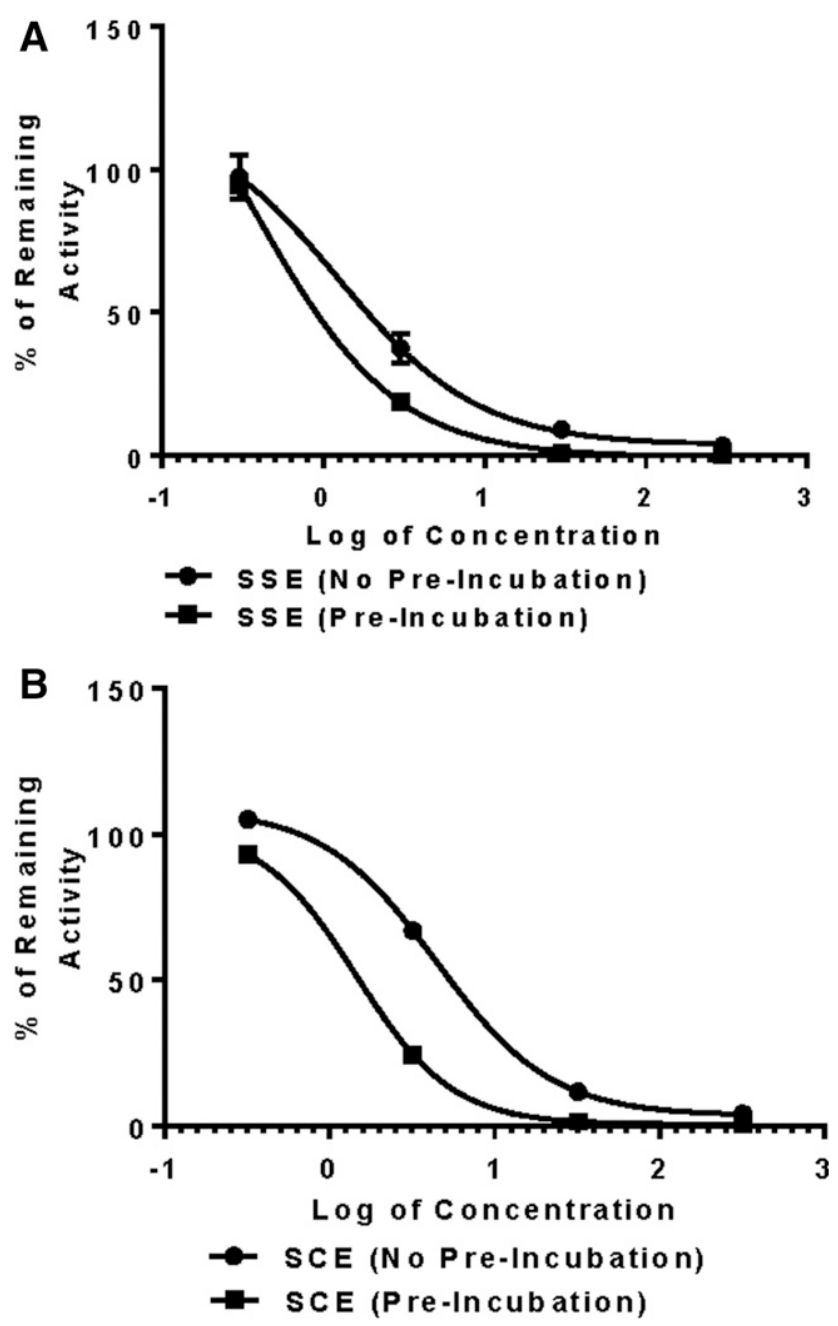

Fig. 2. Inhibition of CYP3A4/5 by SSE (A) or SCE (B), without preincubation (•)

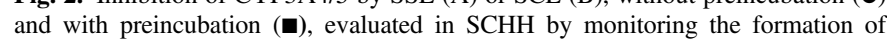
OHMDZ from DZ. SSE no preincubation $\mathrm{IC}_{50}=1.23 \mu \mathrm{g} / \mathrm{ml}, 95 \%$ confidence interval $=0.823-1.85$, goodness of fit $=0.989 ;$ SSE preincubation $\mathrm{IC}_{50}=0.378$ $\mu \mathrm{g} / \mathrm{ml}, 95 \%$ confidence interval $=0.299-0.479$, goodness of fit $=0.999$. SCE no preincubation $\mathrm{IC}_{50}=4.46 \mu \mathrm{g} / \mathrm{ml}, 95 \%$ confidence interval $=4.44-4.48$, goodness of fit $=1.0$; $\mathrm{SCE}$ preincubation $\mathrm{IC}_{50}=1.45 \mu \mathrm{g} / \mathrm{ml}, 95 \%$ confidence interval $=$ $1.44-1.46$, goodness of fit $=1.0$. Data are from a single experiment with treatments performed in triplicate wells. Mean values are reported \pm S.D.

presence of SSE ( $3 \mu \mathrm{g} / \mathrm{ml})$ and SCE $(3.2 \mu \mathrm{g} / \mathrm{ml})$ in SCHH was timedependent. Nonlinear regression analysis of the inhibition plots (with and without preincubation) estimated a $\geq 3$-fold shift in the calculated $\mathrm{IC}_{50}$ values for both Schisandra spp. extracts. The inhibition potency shift of both extracts was statistically significant $(P$ value $\leq 0.05)$, since the calculated $95 \%$ confidence intervals of the $\mathrm{IC}_{50}$ values (with and without preincubation) did not intersect. The observed inhibition potency shift confirmed that both extracts exhibited the potential to inhibit CYP3A4/5 activity in a timedependent manner (Fig. 2).

CYP3A4 mRNA Induction Assessment. Following 72 hours of treatment, CYP3A4 mRNA content was evaluated to assess CYP3A4 induction potential of Schisandra spp. extracts, $\operatorname{SSE}(0.30,3.0,30.0$, and $300 \mu \mathrm{g} / \mathrm{ml})$ and SCE $(0.32,3.2,32$, and $320 \mu \mathrm{g} / \mathrm{ml})$. CYP3A4 mRNA content was induced 8.56-fold and 5.54-fold greater than solvent control in SCHHs treated with RIF $(10 \mu \mathrm{M})$ and SJW extract $(20 \mu \mathrm{g} / \mathrm{ml})$, respectively (Fig. 3). These results demonstrated that SJW, a well studied herbal extract, produced a CYP3A4 induction response that was

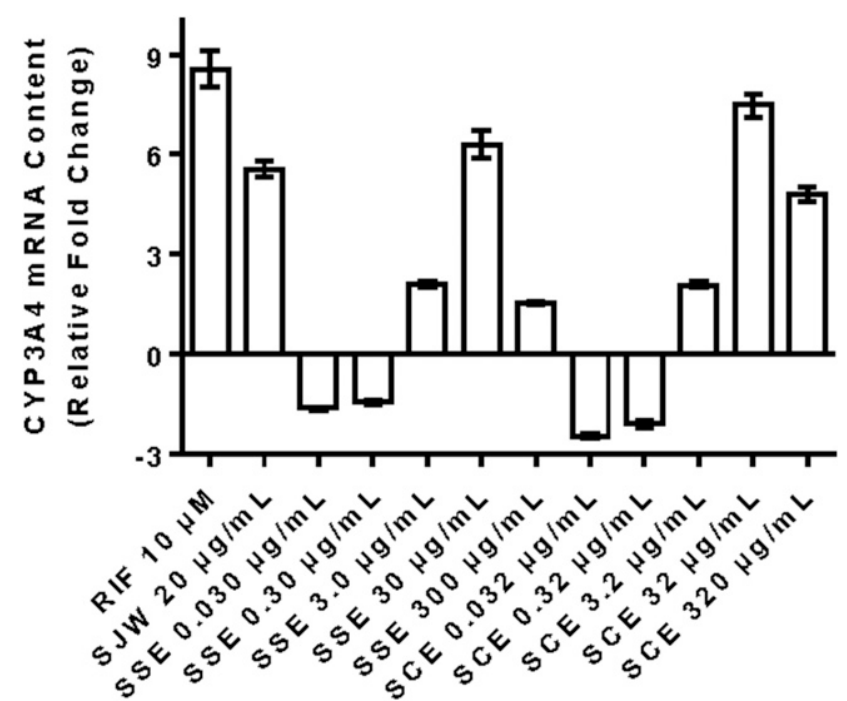

Fig. 3. Relative-fold change of CYP3A4 mRNA content in SCHH following 72 hours of treatment with RIF $(10 \mu \mathrm{M})$, SJW $(20 \mu \mathrm{g} / \mathrm{ml})$, SSE, or SCE. Error bars represent $95 \%$ confidence intervals. Data are from a single experiment with treatments performed in triplicate wells.

$65 \%$ of the response elicited by RIF. These results demonstrated that the SCHH system was sensitive to both pharmaceutical and herbal CYP3A4-type inducers. Dose-related induction of CYP3A4 mRNA content was observed, ranging from 2.09- to 6.27-fold and from 2.08- to 7.48-fold in SCHH exposed to 3.0 and $30 \mu \mathrm{g} / \mathrm{ml} \mathrm{SSE}$ and 3.2 and $32 \mu \mathrm{g} / \mathrm{ml} \mathrm{SCE}$, respectively (Fig. 3). CYP3A4 induction responses provoked by 72 hours of treatment with SSE $(30 \mu \mathrm{g} / \mathrm{ml})$ and SCE $(32 \mu \mathrm{g} / \mathrm{ml})$ were greater than the response produced by SJW extract treatment and were $73 \%$ and $87 \%$ of the RIF response. CYP3A4 induction responses were muted in $\mathrm{SCHH}$ treated with the highest concentrations of SSE and SCE examined (Fig. 3). Loss of ATP content was observed at the highest concentrations of SSE and SCE examined and may explain the reduced CYP3A4 induction response observed under these conditions. Overall, the results demonstrated that both SSE and SCE have the potential to strongly induce CYP3A4 mRNA content in SCHH under the conditions studied.

Intracellular Concentration of Schisandra spp. Constituents. B-CLEAR technology was used to determine the ICC of Schisandra spp. extract constituents, including schisandrin A, gomisin A, and schizandrin, in SCHH following 72 hours of treatment with SSE (30 and $300 \mu \mathrm{g} / \mathrm{ml}$ ) or SCE (30 and $300 \mu \mathrm{g} / \mathrm{ml}$ ). These constituents were chosen because they represent the more abundant lignans found across Schisandra spp. and, therefore, could be used to show that such lignans could accumulate in hepatocytes. ICCs of schisandrin A increased in a dose-related manner in hepatocytes treated with SSE, resulting in concentrations ranging from 28.2 to $1228 \mu \mathrm{M}$ (Fig. 4). Gomisin A and schizandrin were not detected in SCHH lysates treated with SSE at any concentration examined. All three constituents were observed in hepatocytes treated with SCE, where ICCs of gomisin A and schisandrin A increased in a dose-related manner, ranging from 1.51 to $6.40 \mu \mathrm{M}$ and 14.3 to $438 \mu \mathrm{M}$, respectively (Fig. 4). In contrast, schizandrin ICCs were not observed to increase in $\mathrm{SCHH}$ treated with increasing concentrations of SCE. Schisandrin A accumulation was 1.97-fold and 2.8-fold greater in SCHH treated with SSE at 30 and $300-\mu \mathrm{g} / \mathrm{ml}$ dose levels, respectively, compared with SCHH treated with SCE. In general, these data demonstrated that constituents of Schisandra spp. extracts were extensively present in hepatocytes, and each extract examined had a different intracellular concentration profile. 


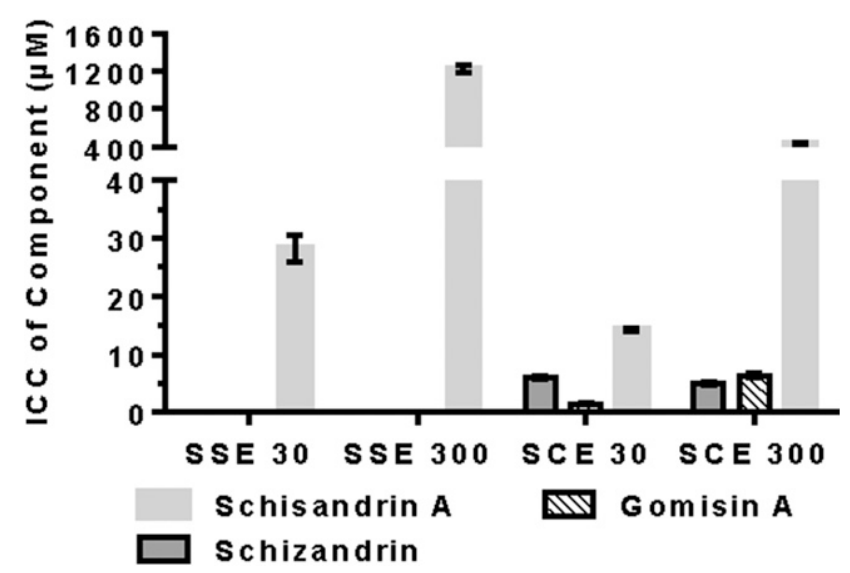

Fig. 4. Intracellular concentrations of schizandrin, gomisin A, and schisandrin A in SCHH following 72 hours of treatment with SSE or SCE. Data from a single experiment with treatments performed in triplicate wells. Mean values reported \pm S.D.

MDZ Intrinsic Clearance. SCHHs were treated for 72 hours prior to monitoring MDZ $(1 \mu \mathrm{M})$ depletion over time to assess SSE (3 or $30 \mu \mathrm{g} / \mathrm{ml}$ ) or SCE (3 or $30 \mu \mathrm{g} / \mathrm{ml}$ ) treatment on intrinsic clearance. Percentage of parent drug remaining versus time profiles (Fig. 5) demonstrated that MDZ intrinsic clearance was clearly increased in SCHHs treated with known CYP3A4 inducers SJW $(20 \mu \mathrm{g} / \mathrm{ml})$ and RIF $(10 \mu \mathrm{M})$. MDZ intrinsic clearance was increased to 2.36-fold or 11.8-fold above solvent control in SCHH treated with SJW or RIF, respectively (Fig. 6A). These results were in agreement with clinical studies that demonstrated a 2.0-fold (Backman et al., 1996) and 24-fold (Wang et al., 2001) increase in the mean MDZ clearance in humans following treatment with SJW or RIF, respectively. In contrast, MDZ intrinsic clearance in SCHH treated with either SSE (3 or $30 \mu \mathrm{g} / \mathrm{ml}$ ) or SCE (3 or $30 \mu \mathrm{g} / \mathrm{ml}$ ) was reduced (Fig. 5), ranging from 0.518- to 0.577 fold and 0.646- to 0.599-fold of solvent control, respectively, consistent with inhibition of metabolism (Fig. 6B). The in vitro results of SSE were in agreement with clinical findings that showed a 0.52 -fold reduction of MDZ clearance across 12 healthy male subjects following a 7-day SSE treatment regimen (Xin et al., 2009). Similar in vitro results were observed for SCE, suggesting that SCE treatment has the potential to cause a similar in vivo effect.

\section{Discussion}

Dietary supplement sales have grown in the United States, reaching over $\$ 6$ billion in 2015 (Smith et al., 2016). Consumer usage data for dietary supplements from 2007 to 2011 found that 64\%-69\% of respondents regularly use dietary supplements beyond simple multivitamins (Dickinson et al., 2014). In conjunction with increased dietary supplement usage, concurrent use with prescription medications is of concern, especially in older adults (Jou and Johnson, 2016). Thus, more routine assessments of herbal ingredients with prescription medications appear warranted.

Although clinical studies are definitive, a clinical strategy is unrealistic for routine HDI assessments. A more realistic approach would be to use a physiologic, relevant in vitro model system, such as SCHH. Using properly characterized hepatocytes (e.g., transporter-certified) under optimized culture conditions, SCHHs are particularly well suited to evaluate complex mixtures for the potential of HDI, as the hepatic whole-cell system maintains drug metabolism (phase I/II) and transporter (uptake/efflux) clearance pathways and vital drug clearance regulatory pathways (CAR/PXR) (Ghibellini et al., 2007; Hewitt et al., 2007; Jackson et al., 2009). Integration of these components into a whole-cell system is necessary in predicting HDI.
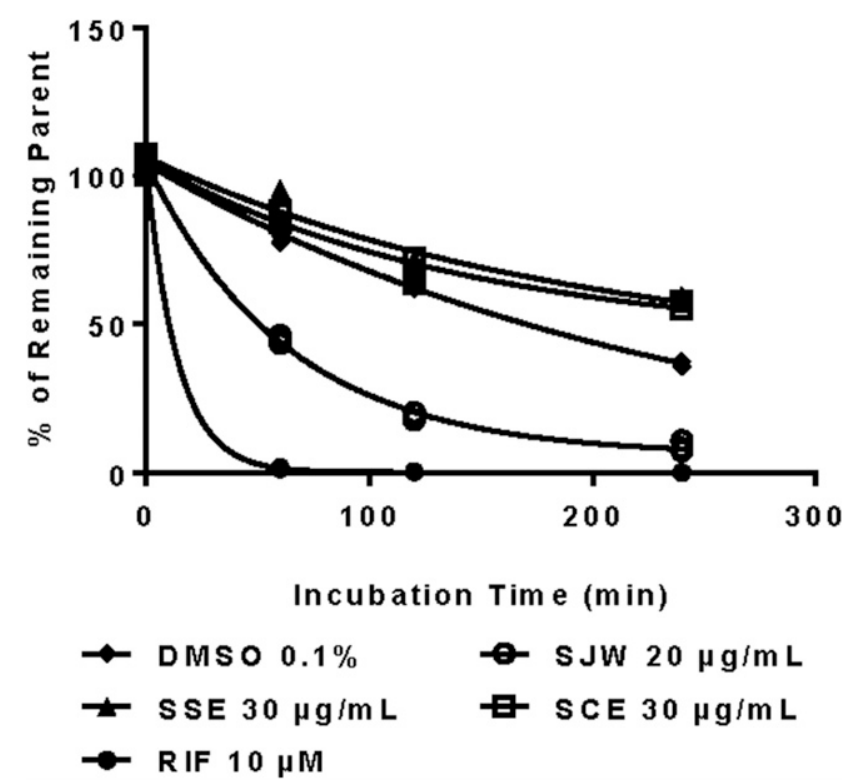

Fig. 5. SCHH were treated with RIF, SJW, SSE, or SCE for 72 hours prior to an MDZ $(1 \mu \mathrm{M})$ incubation of 24 hours, monitoring for depletion of substrate. Disappearance of MDZ from the SCHH system was plotted over time. Data are from a single experiment with treatments performed in triplicate wells. Mean values are reported \pm S.D.

In this study, we evaluated the effectiveness of SCHH to predict in vivo HDI outcomes using an intrinsic clearance approach. Mechanistic inhibition and induction studies of CYP3A4 were performed to further elucidate the drug-interaction mechanisms in $\mathrm{SCHH}$ following exposure to herbal extracts. SSE was used as a model herbal extract with a known MDZ clinical HDI, whereas the closely related and increasingly popular herbal dietary supplement extract SCE, with an unknown clinical MDZ HDI profile, was evaluated in parallel.

Mechanistic inhibition studies in SCHH demonstrated that both SSE and SCE reduced the rate of OHMDZ formation in a dose-dependent manner with and without preincubation. Nonlinear regression analysis of the inhibition curves demonstrated that the inhibition potency $\left(\mathrm{IC}_{50}\right)$ of both Schisandra spp. extracts increased $\geq 3$-fold with preincubation, indicative of time-dependent inhibition. Toxicity did not appear to be the reason for the loss of OHMDZ formation since ATP content remained unchanged following 24-hour exposure to $30 \mu \mathrm{g} / \mathrm{ml} \mathrm{SSE}$ and $32 \mu \mathrm{g} / \mathrm{ml}$ SCE. At these concentrations, inhibition of OHMDZ formation reached maximum effect, reducing activity to $99 \%$ and $98 \%$ of control, respectively, following a 60 -minute preincubation. Taken together, these results demonstrated that loss of CYP3A4/5 activity was not attributable to cytotoxicity but was the result of enzyme time-dependent inhibition.

Using a standard in vitro approach recommended by the regulatory agencies (European Medicines Agency, 2012; Food and Drug Administration, 2012), induction of CYP3A4 mRNA was evaluated using transporter-certified hepatocytes in a sandwich-culture format following 72 hours of exposure to increasing concentrations of either Schisandra spp. extract. Interestingly, robust dose-related induction of CYP3A4 mRNA was observed in SCHH treated with either Schisandra spp. extract across the concentrations examined. A maximum CYP3A4 mRNA response 6.27-fold and 7.48-fold greater than vehicle control was observed in SCHH treated with $30 \mu \mathrm{g} / \mathrm{ml} \mathrm{SSE}$ or $32 \mu \mathrm{g} / \mathrm{ml} \mathrm{SCE}$, respectively. Also, the maximum CYP3A4 induction observed in response to SSE or SCE was equivalent (113\% and $135 \%$ of SJW) or almost equal ( $73.2 \%$ and $87.4 \%$ of RIF) to induction responses of known CYP3A4 inducers SJW and RIF. An induction response of $20 \%-40 \%$ of 

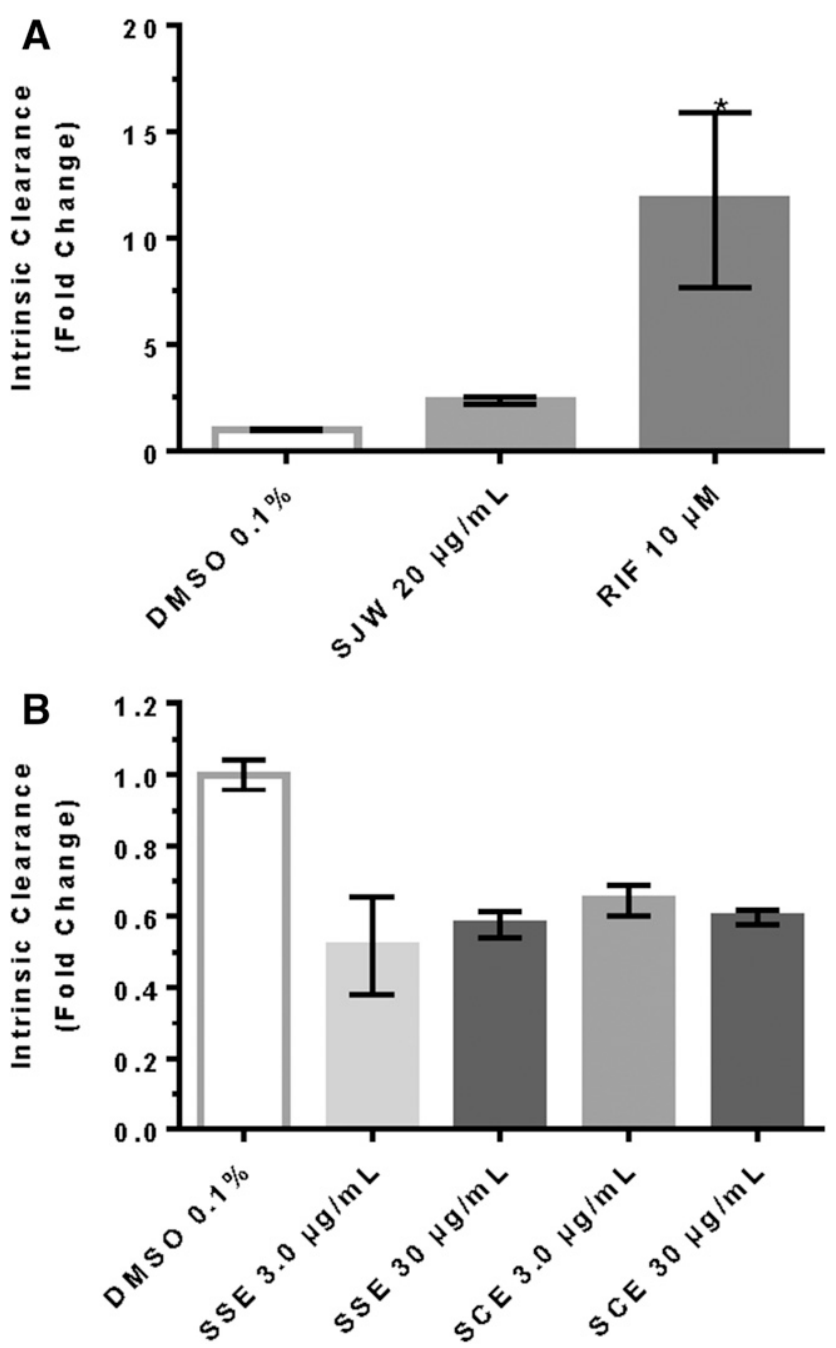

Fig. 6. Intrinsic clearance of MDZ was calculated from linear regression analysis of the percentage of parent remaining (log transformed) versus time profile to estimate the elimination rate. MDZ intrinsic clearance in SCHH treated with SJW (A), RIF (A), SSE (B), or SCE (B) for 72 hour. $* P$ value $\leq 0.05$ when compared with solvent control. Data are from a single experiment with treatments performed in triplicate wells. Mean values reported \pm S.D.

positive control has previously been proposed as demonstrating clinically relevant induction potential (Bjornsson et al., 2003; Food and Drug Administration, 2006; Fahmi et al., 2010). Induction of CYP3A4 mRNA was reduced from maximum in SCHH treated at the highest concentrations of SSE or SCE evaluated. Following 72 hours of treatment at these high concentrations only, ATP content was significantly ( $P$ value $\geq 0.05$ ) reduced, suggesting that the loss of CYP3A4 induction may have been the result of hepatotoxicity.

Our approach, using a fully integrated whole-cell system, demonstrated that both Schisandra spp. extracts significantly inhibited and induced CYP3A4 metabolism, a drug clearance pathway extensively involved in the clearance of $\geq 30 \%$ of clinically prescribed medications (Zanger and Schwab, 2013). These results suggested that both Schisandra spp. extracts have the potential to elicit an HDI within a major drug clearance pathway; however, the net effect of Schisandra spp. extract exposure was difficult to estimate from these separate mechanistic studies. To evaluate the HDI net effect and predict an overall clinical effect, further experiments were performed using the same whole-cell system treated for 72 hours with either SSE or SCE (3.0 and $30 \mu \mathrm{g} / \mathrm{ml}$ ) prior to monitoring the intrinsic clearance of MDZ.
Demonstrating that this system was responding appropriately, the positive controls, SJW and RIF, increased intrinsic clearance of MDZ 2.36-fold and 11.8-fold above solvent control, respectively. These data were consistent with reported clinical MDZ drug interactions. In clinical drug-interaction studies, SJW and RIF increased MDZ clearance 2.0fold and 24-fold, correspondingly (Backman et al., 1996; Wang et al., 2001). These results demonstrated that an intrinsic clearance approach in SCHH using a sensitive probe substrate (MDZ) to assess the drug interaction net effect (e.g., induction) and the relative strength of the interaction was feasible. These in vitro results were in good agreement with clinical-interaction studies of well known perpetrators of in vivo drug interactions, SJW and RIF. Using our integrated SCHH system, intrinsic clearance of MDZ was reduced to 0.518 -fold and 0.577 -fold of solvent control treated with 3.0 and $30 \mu \mathrm{g} / \mathrm{ml} \mathrm{SSE}$, respectively. These results were in remarkable agreement with the previously mentioned clinical HDI study that demonstrated MDZ clearance was reduced 0.52fold across 12 healthy volunteers treated with SSE twice daily for 7 days. Similarly, for the SCE extract, which had not been evaluated clinically, intrinsic clearance of MDZ was reduced to 0.646-fold and 0.599-fold of solvent control in SCHH treated with 3.0 and $30 \mu \mathrm{g} / \mathrm{ml} \mathrm{SCE}$, respectively. Although the changes in MDZ clearance were in close agreement with the clinical studies and support this screening approach, true translation to clinical data would require additional experiments, incorporation of other organ effects, and potentially in silico modeling efforts. The inhibition mechanism studies described earlier for both Schisandra spp. extracts demonstrated dose-related inhibition, with maximum inhibition achieved at $\sim 30 \mu \mathrm{g} / \mathrm{ml}$; however, no dose-related effects were observed with MDZ intrinsic clearance. The lack of dosedependent inhibition of MDZ intrinsic clearance following 72 hours of either Schisandra spp. extract treatments (e.g., 3 and $30 \mu \mathrm{g} / \mathrm{ml}$ ) may have been due to CYP3A4 induction. In support of this conclusion, mechanistic induction studies demonstrated that SSE and SCE maximally induced CYP3A4 mRNA at $\sim 30 \mu \mathrm{g} / \mathrm{ml}$.

SCHHs cultured under optimized conditions have active transport and adaptive response mechanisms necessary to generate physiologically relevant intracellular concentrations. Thus, intracellular concentrations were determined for three of the more abundant constituents across Schisandra spp. extracts in our optimized cultures of SCHH following 72 hours of treatment with SSE or SCE at 30 and $300 \mu \mathrm{g} / \mathrm{ml}$. All three constituents were observed in hepatocytes treated with SCE. Schisandrin A intracellular concentrations were 2.8-fold greater in SCHH treated with SSE at the $300-\mu \mathrm{g} / \mathrm{ml}$ dose level than in that treated with SCE. Interestingly, and consistent with these concentration differences, SSE was demonstrated to be a $\sim 3.8$-fold more-potent inhibitor of OHMDZ formation than SCE in the mechanistic inhibition studies. Intracellular concentration differences may explain these findings. In microsomal studies evaluating individual SCE constituents, the gomisins (A, B, G, $\mathrm{C}$, and $\mathrm{N}$ ) were reported to be more-potent inhibitors of CYP3A4 activity than schisandrins (Iwata et al., 2004). HDI assessment strategies that interrogate each component separately for interaction potential may not account for synergistic effects among constituents; hence, a more integrative approach is required to evaluate complex mixtures and reproduce net drug-interaction effects.

In summary, we demonstrated that an intrinsic clearance approach in sandwich-cultured transporter-certified human hepatocytes, with integrated metabolism and transport functionality, was feasible to evaluate HDI net effect and the relative strength of the HDI. We believe this neteffect approach is an appropriate strategy to screen complex mixtures for drug interactions. It should be noted that additive or synergistic effects between mixture components were not explicitly evaluated, and additional studies would be required to explore those effects. The approach was remarkably consistent with the in vivo HDI effect 
observed in the clinic for two herbal extracts, SSE and SJW. The evaluation of SCE predicted an in vivo HDI with MDZ similar to that observed with the closely related SSE. Kinetic parameters $\left(\mathrm{K}_{\mathrm{i}}, \mathrm{KI}, \mathrm{IC}_{50}\right.$, $\mathrm{EC}_{50}$ ) from mechanistic studies have been successful in evaluating druginteraction potential; however, kinetic parameters are difficult to directly translate to an in vivo net effect. Although our results were in good agreement with clinical findings, improvements to the study design should be considered, including the use of multiple donors for intrinsic clearance studies and developing an in vitro assay to predict herbal extract bioavailability from gut absorption. Although many drug interactions occur in the hepatocyte, the gut is an additional site of interaction that has to be considered to fully evaluate the net effect and strength of drug interactions. Here, we present a strategy to efficiently predict the potential for clinically relevant HDI by using a fully integrated in vitro system that maintains key hepatic processes important to evaluating drug interactions and can provide intracellular concentrations of constituents to further elucidate mechanistic details.

\section{Authorship Contributions}

Participated in research design: Jackson, Black, Brouwer, Roe.

Conducted experiments: Jackson, Freeman, Friley.

Performed data analysis: Jackson, Freeman, Friley, Black, Brouwer, Roe.

Wrote or contributed to the writing of the manuscript: Jackson, Freeman, Herman, Friley, Black, Brouwer, Roe.

\section{References}

Backman JT, Olkkola KT, and Neuvonen PJ (1996) Rifampin drastically reduces plasma concentrations and effects of oral midazolam. Clin Pharmacol Ther 59:7-13.

Bjornsson TD, Callaghan JT, Einolf HJ, Fischer V, Gan L, Grimm S, Kao J, King SP, Miwa G, N L, et al.; Pharmaceutical Research and Manufacturers of America Drug Metabolism/Clinical Pharmacology Technical Working Groups (2003) The conduct of in vitro and in vivo drug-drug interaction studies: a PhRMA perspective. J Clin Pharmacol 43:443-469.

Brown HS, Chadwick A, and Houston JB (2007) Use of isolated hepatocyte preparations for cytochrome P450 inhibition studies: comparison with microsomes for Ki determination. Drug Metab Dispos 35:2119-2126.

Chandra P and Brouwer KL (2004) The complexities of hepatic drug transport: current knowledge and emerging concepts. Pharm Res 21:719-735.

Davies B and Morris T (1993) Physiological parameters in laboratory animals and humans. Pharm Res 10:1093-1095.

Dickinson A, Blatman J, El-Dash N, and Franco JC (2014) Consumer usage and reasons for using dietary supplements: report of a series of surveys. J Am Coll Nutr 33:176-182.

Essigmann JM, Croy RG, Nadzan AM, Busby WF Jr, Reinhold VN, Büchi G, and Wogan GN (1977) Structural identification of the major DNA adduct formed by aflatoxin B1 in vitro. Proc Natl Acad Sci USA 74:1870-1874.

European Medicines Agency (2012) Guideline on the Investigation of Drug Interactions, European Medicines Agency, London.

Fahmi OA, Kish M, Boldt S, and Obach RS (2010) Cytochrome P450 3A4 mRNA is a more reliable marker than CYP3A4 activity for detecting pregnane $\mathrm{X}$ receptor-activated induction of drug-metabolizing enzymes. Drug Metab Dispos 38:1605-1611.
Food and Drug Administration (2006) Draft Guidance for Industry - Drug Interaction Studies: Study Design, Data Analysis, and Implications for Dosing and Labeling, Food and Drug Administration Publication, Silver Spring, MD.

Food and Drug Administration (2012) Draft Guidance for Industry - Drug Interaction Studies: Study Design, Data Analysis, and Implications for Dosing and Labeling, Food and Drug Administration Publication, Silver Spring, MD.

Ghibellini G, Vasist LS, Leslie EM, Heizer WD, Kowalsky RJ, Calvo BF, and Brouwer KLR (2007) In vitro-in vivo correlation of hepatobiliary drug clearance in humans. Clin Pharmacol Ther 81:406-413.

Gurley BJ (2012) Pharmacokinetic herb-drug interactions (part 1): origins, mechanisms, and the impact of botanical dietary supplements. Planta Med 78:1478-1489.

Hewitt NJ, Lechón MJG, Houston JB, Hallifax D, Brown HS, Maurel P, Kenna JG, Gustavsson L, Lohmann C, Skonberg C, et al. (2007) Primary hepatocytes: current understanding of the regulation of metabolic enzymes and transporter proteins, and pharmaceutical practice for the use of hepatocytes in metabolism, enzyme induction, transporter, clearance, and hepatotoxicity studies. Drug Metab Rev 39:159-234.

Iwata H, Tezuka Y, Usia T, Kadota S, Hiratsuka A, and Watabe T (2004) Inhibition of human liver microsomal CYP3A4 and CYP2D6 by extracts from 78 herbal medicines. J Trad Med 21:42-50. Jackson JP, Kabirov KK, Kapetanovic IM, and Lyubimov A (2009) In vitro assessment of P450 induction potential of novel chemopreventive agents SR13668, 9-cis-UAB30, and pentamethychromanol in primary cultures of human hepatocytes. Chem Biol Interact 179:263-272.

Jou J and Johnson PJ (2016) Nondisclosure of complementary and alternative medicine use to primary care physicians: findings from the 2012 National Health Interview Survey. JAMA Intern Med 176:545-546.

Lee JK and Brouwer KR (2010) Determination of intracellular volume of rat and human sandwichcultured hepatocytes (Abstract). The Toxicologist Toxicol Sci 114 (Suppl):339.

Liu X, Chism JP, LeCluyse EL, Brouwer KR, and Brouwer KLR (1999) Correlation of biliary excretion in sandwich-cultured rat hepatocytes and in vivo in rats. Drug Metab Dispos 27 637-644.

Marion TL, Perry CH, St Claire RL, III, and Brouwer KLR (2012) Endogenous bile acid disposition in rat and human sandwich-cultured hepatocytes. Toxicol Appl Pharmacol 261:1-9.

McGinnity DF, Tucker J, Trigg S, and Riley RJ (2005) Prediction of CYP2C9-mediated drug-drug interactions: a comparison using data from recombinant enzymes and human hepatocytes. Drug Metab Dispos 33:1700-1707.

Qin XL, Bi HC, Wang XD, Li JL, Wang Y, Xue XP, Chen X, Wang CX, Xu J, Wang YT, et al. (2010) Mechanistic understanding of the different effects of Wuzhi Tablet (Schisandra sphenanthera extract) on the absorption and first-pass intestinal and hepatic metabolism of Tacrolimus (FK506). Int J Pharm 389:114-121.

Roe AL, Paine MF, Gurley BJ, Brouwer KR, Jordan S, and Griffiths JC (2016) Assessing natural product-drug interactions: an end-to-end safety framework. Regul Toxicol Pharmacol 76:1-6.

Smith T, Kawa K, Eckl V, and Johnson J (2016) Sales of herbal dietary supplements in US increased 7.5\% in 2015. HerbalGram 111:67-73.

Sprouse AA and van Breemen RB (2016) Pharmacokinetic interactions between drugs and botanical dietary supplements. Drug Metab Dispos 44:162-171.

Wang Z, Gorski JC, Hamman MA, Huang SM, Lesko LJ, and Hall SD (2001) The effects of S John's wort (Hypericum perforatum) on human cytochrome P450 activity. Clin Pharmacol Ther 70:317-326.

Xin HW, Wu XC, Li Q, Yu AR, Zhu M, Shen Y, Su D, and Xiong L (2007) Effects of Schisandra sphenanthera extract on the pharmacokinetics of tacrolimus in healthy volunteers. Br J Clin Pharmacol 64:469-475.

Xin HW, Wu XC, Li Q, Yu AR, and Xiong L (2009) Effects of Schisandra sphenanthera extract on the pharmacokinetics of midazolam in healthy volunteers. Br J Clin Pharmacol 67:541-546.

Zanger UM and Schwab M (2013) Cytochrome P450 enzymes in drug metabolism: regulation of gene expression, enzyme activities, and impact of genetic variation. Pharmacol Ther 138: 103-141.

Address correspondence to: Jonathan P. Jackson, Qualyst Transporter Solutions, 2810 Meridian Parkway, Suite 100, Durham, NC 27713. E-mail: JonathanJackson@ qualyst.com 